\title{
Pierre Joseph - Exposition, proposition, délégation : une pratique artistique à l'ère de la signature multiple
}

Entretien avec Nicolas Donin et Christophe Kihm

\section{Nicolas Donin et Christophe Kihm}

\section{OpenEdition}

\section{Journals}

Édition électronique

URL : http://journals.openedition.org/genesis/1554

DOI : 10.4000/genesis. 1554

ISSN : 2268-1590

Éditeur :

Presses universitaires de Paris Sorbonne (PUPS), Société internationale de génétique artistique littéraire et scientifique (SIGALES)

\section{Édition imprimée}

Date de publication : 27 novembre 2015

Pagination : 131-143

ISBN : 9791023105049

ISSN : 1167-5101

\section{Référence électronique}

Nicolas Donin et Christophe Kihm, «Pierre Joseph - Exposition, proposition, délégation : une pratique artistique à l'ère de la signature multiple », Genesis [En ligne], 41 | 2015, mis en ligne le 21 avril 2017, consulté le 17 mai 2019. URL : http://journals.openedition.org/genesis/1554 ; DOI : 10.4000/ genesis. 1554 


\title{
Pierre Joseph \\ Exposition, proposition, délégation : une pratique artistique à l'ère de la signature multiple
}

\author{
Entretien avec Nicolas Donin et Christophe Kihm
}

Le travail artistique de Pierre Joseph (1965) fut tout d'abord associé à un groupe d'artistes ayant développé leurs pratiques, à partir de la fin des années quatre-vingt, à travers des protocoles et des formes renouvelées de la collaboration. Dominique Gonzalez-Foerster (1965), Philippe Parreno (1964), Philippe Perrin (1964) et Bernard Joisten (1964) furent les principaux acteurs de ces échanges, auxquels ont pu se joindre, plus tard, d'autres artistes, notamment Pierre Huyghe (1962). Cette génération a investi des questions relatives à la signature et par conséquent au statut des œuvres d'art selon une perspective prenant en compte les formes de l'exposition. Le nouage de ces deux préoccupations a longtemps identifié leurs pratiques collectives, regroupées sous l'appellation d' "esthétique relationnelle " par Nicolas Bourriaud, sans pour autant donner lieu à un examen détaillé de leur histoire ni à une approche concrète de leurs protocoles et procédures. Alors que pour beaucoup de ces acteurs, ces collaborations se sont faites plus rares, Pierre Joseph a poursuivi dans cette voie, y impliquant aussi bien des écrivains que des étudiants en écoles d'art, de jeunes artistes ou des personnes étrangères au milieu de l'art-dans d'autres projets, mais surtout sur d'autres terrains. Car à la fin des années quatre-vingt-dix et à la faveur d' une résidence au Japon, Pierre Joseph a réorienté sa pratique autour d' une enquête singulière sur les compétences, les connaissances et les savoirs, s'intéressant aux conditions de leur apprentissage, mais surtout aux vides ou aux manques qui les constituent, auxquels il cherche à donner forme.

La première présentation publique du travail de Pierre Joseph fut une exposition coréalisée avec Bernard Joisten et Philippe Parreno, "19\&\& », au Magasin, centre national d'art contemporain, à Grenoble en 1988. Les artistes y présentaient Siberia, un container isotherme posé à l'extérieur du Magasin et contenant un ensemble d'images rétroéclairées se rapportant, dans leur matière et dans leur contenu, aux nouvelles technologies d'alors. Les trois artistes enchaînèrent sur Composit (Crédac, Ivry-sur-Seine, 1988), où s'entremêlaient trois propositions autour du traitement (notamment numérique) de l'image d'actualité, puis, rejoints par Dominique Gonzalez-Foerster, Ozone (commande du FRAC Corse). «Ni marque, ni produit », Ozone était moins une auvre ou une exposition qu' un "programme à applications multiples créé et géré par quatre personnes ${ }^{1} »$ autour des concepts et représentations de l'écologie devenue, ou devenant, un enjeu social global.

À partir de 1991, Pierre Joseph conçoit une série de Personnages à réactiver : le cow-boy, la fée, Don Quichotte, Cupidon, le motard, le noyé, Superman, Catwoman, Blanche-Neige, les "voleurs de couleur » d'une fameuse publicité Kodak, le paintballer, le policier... Chaque présentation de l' ouvre dans un lieu d'exposition consiste en la présence, au cours du vernissage, d'une personne dont l'accoutrement et le comportement (ou la posture, si le personnage est réputé inanimé) correspondent à un programme minimal défini par l'artiste. Dès le lendemain, la présence physique du personnage est remplacée par une image photographique. Pierre Joseph n'a pas poursuivi cette série qui l'a rendu célèbre, mais participe toujours au devenir des auvres lors de leurs réactivations.

Parmi les jalons de son travail au cours des années deux mille, mentionnons Parlez-moi, dictionnaire des mots dont j'ai l'usage et dont je comprends le sens (1999), la série des plans et dessins exécutés de mémoire par l'artiste (Mon plan du plan du métro de Paris, 2000) ou par d'autres (Atlas, 2004-2006), la Table sans nom (2004), l'exposition "Rétrospective » au sein de la biennale de Lyon (2007), L’École de Stéphanie (2006-2015), Pourquoi tout n’a-t-il pas déjà disparu ? (2010)..

L'entretien a lieu dans l'atelier parisien de Pierre Joseph le 9 septembre 2014.

1. Notice d'Ozone d'après Pierre Joseph, Oui non peut-être, Dijon/Paris, Les Presses du Réel et M/M, 2011, p. 136. 


\section{Artiste(s) et commissaire(s)}

Nicolas Donin et Christophe Kihm - Pouvez-vous revenir sur votre proposition pour la biennale de Lyon de 2007, dont les commissaires étaient Stéphanie Moisdon et Hans Ulrich Obrist? Le cadre de l' exposition se donnait sous la forme d' un jeu qui impliquait la participation des artistes, et auquel vous avez répondu par un protocole de travail adressé à d'autres artistes, prenant en compte certains de vos travaux, qui eux-mêmes avaient pu engager des collaborations. Peut-on revenir sur cet emboîtement? Comment répondez-vous à la sollicitation des commissaires?

Pierre Joseph - Stéphanie Moisdon et Hans Ulrich Obrist font une proposition d'intervention. C'est de Stéphanie Moisdon qu'émane la demande. Elle consiste à organiser une situation d'exposition de jeunes artistes français, sachant que je suis professeur aux beaux-arts de Montpellier, que je suis intéressé par l'arrivée de jeunes générations et par les réponses qu'elles peuvent apporter à une situation (d'exposition). Stéphanie me demande de faire un travail de repérage. Le principe de cette biennale de Lyon était double : un regard sur le début des années deux mille et un jeu. Je me suis dit que j'allais proposer certaines parties de mon travail à une relecture, à une interprétation, à une réactivation, à une transformation, auprès de très jeunes artistes. J'ai proposé cette situation. J'ai envoyé un email à une sélection d'artistes que j'avais repérés, dont j'avais vu le travail dans différentes expositions, souvent à partir de la lecture des articles des Inrockuptibles sur leurs expositions. J'avais ces journaux et je les ai repris, puis à partir d'autres éléments que j'avais en mémoire, $\mathrm{j}$ 'ai opéré une première sélection de jeunes artistes à qui j'ai envoyé une lettre de protocole, pour leur dire que je les invitais à participer à la biennale de Lyon, mais aussi que je les invitais à prolonger et à réactiver une partie de mon travail. Je voyais dans leurs travaux des prolongements, des survivances, des transformations de choses que moi, ou un ensemble de personnes autour de moi, avions posées.

Vous vous adressez à des gens que vous n'avez jamais rencontrés, auxquels vous demandez quelque chose de très particulier. En quels termes formulez-vous cette demande?
Je leur dis que j'entrevois dans leur travail une continuité par rapport à des questions que j'ai pu me poser précédemment.

Proposez-vous une articulation entre ce qu'ils vont faire et ce que vous avez fait?

Non, je leur demande une proposition. Et à partir de cette proposition, j'envisage quelque chose qui revient sur mon travail. C'est le jeu. À partir de l'énoncé contenu dans ma demande, leur travail se déploie en conservant son autonomie. Je ne fais qu'organiser un passage.

Avez-vous refusé des propositions ou avez-vous tout accepté?

Je n'ai rien refusé.

Comment intervenez-vous dans l' exposition de ces propositions? Est-ce vous qui organisez l'espace que l'on vous a confié ? Et comment procédez-vous?

J'ai fait un écran avec un cartel mouvant, au sein duquel les noms des artistes changeaient alors que le texte restait le même ${ }^{2}$, dans l'entrée (fig. 1). Une fois passé ce sas, on entrait dans une exposition classique, quoique plongée dans le noir, avec des focus lumineux sur les œuvres. J'ai pensé l'espace de manière un peu exponentielle. On partait de salles très serrées, avec Benoît Maire, Benoît Broisat, et plus on avançait plus les salles étaient grandes, jusqu'à l'avion de Fabien Giraud et Raphaël Siboni. J'avais fait ce plan d'un espace qui s'ouvrait au préalable, ensuite j'ai choisi l'emplacement en fonction des propositions des artistes.

2. «Rétrospective est une exposition collective qui fait rejouer certains de mes travaux à des artistes apparus récemment sur la scène artistique française. J'ai demandé par exemple à [NOM DE L'ARTISTE] ce qu'il avait pu retenir de l'art que je produis et ce qu'il en connaissait. Et de chercher avec lui la part de son travail qui reconnaît au mien un antécédent ou une forme de filiation. Nous nous sommes entendus sur les notions de [SÉRIE DE NOTIONS] communes à nos deux approches. Son intérêt s'est alors porté sur l'œuvre [TITRE] [...]. » 


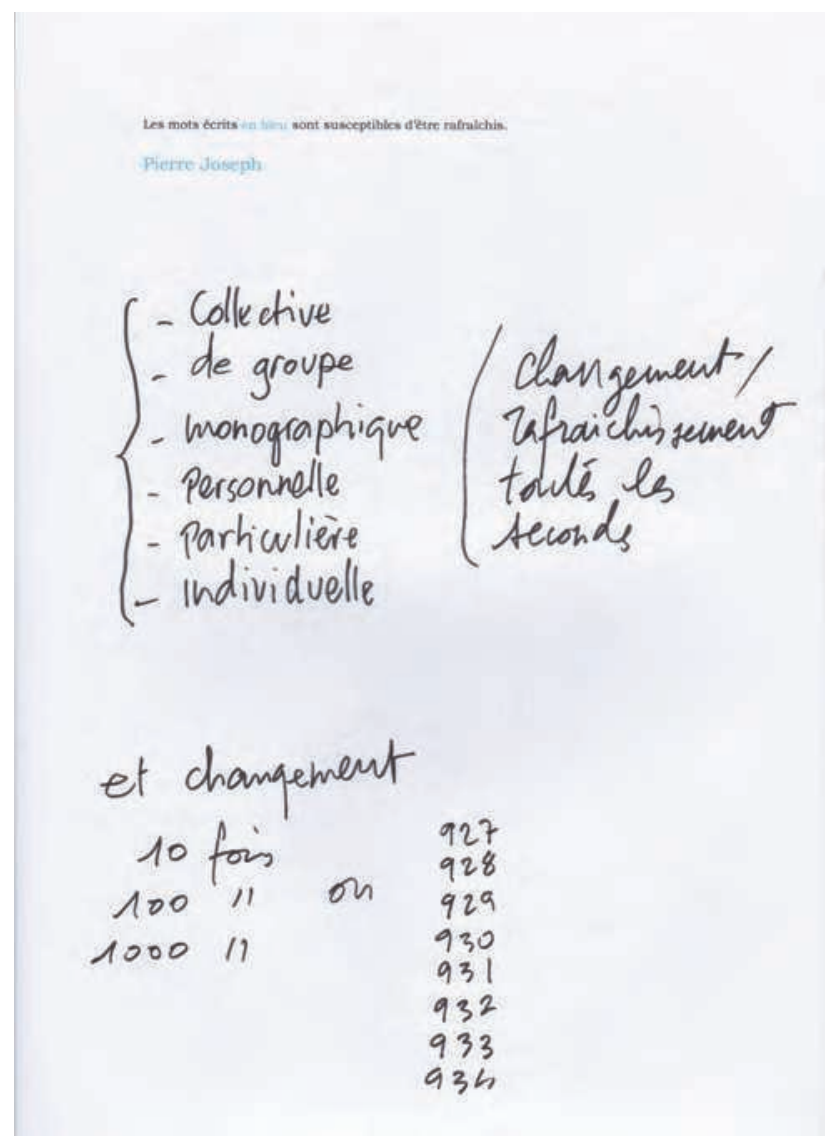

Fig. 1 : Pierre Joseph, Brouillon partiel du cartel animé pour l'entrée de l'espace d'exposition de Rétrospective, 2007

Avez-vous discuté de ce plan avec les commissaires?

Non, je les ai montrés à Stéphanie, mais surtout à Thierry Prat et Thierry Raspail [directeurs artistiques de la biennale de Lyon, NDLR], qui sont davantage impliqués dans les questions matérielles d'organisation, de découpage de l'espace.

Dans cette exposition, on cherchait l'aspect rétrospectif de cette proposition d'ensemble, sans être certain de le trouver. Tout demeurait flottant, ce qui était certainement voulu...

Je lançais une proposition sans aucune certitude quant à la réponse que j'allais obtenir. C'était le principe. Tous les artistes n'ont pas nécessairement joué le jeu. Et puis, ce qui m'intéressait, c'était l'écriture du texte, de ce texte qui racontait une histoire, à partir de ce sentiment qu'aujourd'hui on consomme les artistes. On change les noms, on conserve quelques phrases...

Ce texte est-il dérivé d'autres textes? A-t-il un précédent?

Ça commence par une version un peu plus simple, envoyée par email. Il est retravaillé ensuite pour le catalogue, enfin pour le cartel de l'exposition. Il y a plusieurs versions, dont la première est, je crois, un SMS... Et je ne sais pas quelle est la version la plus intéressante ; au fond, peut-être le SMS de début.

Le procédé de la biennale de Lyon fonctionnait par délégation successive : des directeurs nomment deux commissaires, qui font des propositions à des artistes qui, euxmêmes, sont amenés à faire des propositions à travers un protocole assimilé à un jeu.

Comme nous avions déjà beaucoup travaillé ensemble, Stéphanie savait très bien ce que je pouvais proposer. Et c'est un moment où se pose la question du passage d'une génération à une autre, de continuités, d'incompréhensions, de ruptures... Et même de réalité de ce passage. Ce sont des choses que j'ai voulu mettre en perspective. Je sollicite des gens à qui je demande de se rattacher à moi, pour mieux qu'ils s'en détachent... Et c'est uniquement parce qu' on me propose ce cadre que je le fais, car je ne me vois aucunement associé à une sorte de descendance ou de filiation dans l'art. C'est vraiment de l'ordre du jeu.

\section{Comment avez-vous choisi ce titre, «Rétrospective»?}

C'était comme afficher, de manière un peu provocatrice, l'ambition du projet. Comment imaginer une rétrospective de son travail où le travail ne serait pas présent mais qui montre justement le fait que l'on est passé à autre chose. Le titre existait dès l'adresse aux jeunes artistes, ce qui a bloqué deux d'entre eux. C'est le jeu de la signature, tel qu'on l'a vu évoluer jusqu'à aujourd'hui. 


\section{Premiers projets collaboratifs (1988-1990)}

De quelle manière se sont mises en place les collaborations vous associant à d'autres artistes? Comment vous êtes-vous accordés pour engager ce type de travail à plusieurs?

Tout commence à l'école des beaux-arts de Grenoble. Je suis dans une promotion, Philippe Perrin dans une autre, et ainsi de suite pour Philippe Parreno, Dominique Gonzalez-Foerster et Bernard Joisten. Mais nous nous retrouvons sur des intérêts, on regarde le travail de l'autre, on parle du travail de l'un à tel autre, des groupes se créent. Les professeurs, Ange Leccia ou Jean-Luc Vilmouth, vous disent d'aller voir tel travail ou telle personne. Les choses s'agglomèrent aussi de cette façon et on se retrouve dans des communautés d'intérêts, des communautés affectives, et on commence à échanger. Ensuite, les choses deviennent un peu plus concrètes. Je me souviens d'une petite discussion dans une salle de classe avec Dominique Gonzalez-Foerster, où nous disions que nous avions envie de voir autrement les choses, de voir d'autres choses et de voir de l'art autrement. C'était un désir, de l'envie, mais il n'y avait pas d'objet. Nous n'étions plus intéressés par ce que nous étions en train de voir : on voulait autre chose, mais on ne savait pas quoi. Dominique Gonzalez-Foerster, qui était la plus avancée dans les années d'études (trois années devant moi), faisait des choses un peu bizarres. Par exemple, elle s'était installée au dernier étage des Beaux-arts où elle avait collé un poster sur toutes les baies vitrées. On arrivait ainsi dans une pièce noire, mais on voyait New York. Elle avait invité des gens, nous avions mangé et bu un verre et, pour finir, elle avait ouvert les fenêtres et l'on revenait à la réalité. C'était magnifique. Elle avait aussi suspendu dans l'espace un morceau de bois, sur une chaîne, articulé par une sorte de charnière, qui fonctionnait comme un instrument de mesure de l'espace. Je crois qu'elle avait appelé cela un «instrument d'art», ou une « mesure pour l'art ». Élément fondateur pour beaucoup de choses ensuite, elle avait peint une sorte d'alcôve avec une couleur très simple et, avec un lettrage adhésif, elle avait écrit une série de noms propres qui correspondaient à un certain nombre de choix dans l'actualité culturelle :
David Lynch, Jeff Koons, quelques noms de choses emblématiques du moment. J'avais l'impression qu'on passait à autre chose par rapport au discours des professeurs. J'étais plus jeune et j'avais envie d'aller voir de ce côtélà. J'avais rencontré Parreno, Joisten, et ça a commencé à se mettre en place.

Beaucoup d'affinités électives, qui se font dans ce genre de circonstances, ne mènent pas à concevoir des expositions et des collaborations sur une durée significative. Vous n'aviez pas nécessairement de modèles pour ce type de travaux à plusieurs.

Nous n'avions pas de modèles. Si l'on s'en tient vraiment aux faits : le Magasin ouvrait à Grenoble sous la direction de Jacques Guillot, créant une nouvelle situation. Le lieu avait demandé à des étudiants de l'école s'ils souhaitaient participer à des montages, des animations, etc. Ce qui offrait l'opportunité de rencontrer des artistes : Buren lors de l'ouverture du lieu, Sol LeWitt... C'était formidable et cela nous permettait de nous confronter à une situation professionnelle. Nous ne restions pas dans l'école, avec des professeurs qui bien évidemment nous montraient déjà qu'il y avait un ailleurs, mais avec cette structure, qui avait une envergure internationale, qui invitait de grands artistes. Ensuite, a ouvert l'École du Magasin, dont la vocation était de former de jeunes curateurs et médiateurs, et Dominique Gonzalez-Foerster, sortant de l'école des beaux-arts, a décidé d'intégrer sa formation en un an, en même temps qu'Esther Schipper et d'autres acteurs qui sont devenus galeristes. Ils ont décidé de faire une exposition de fin d'année et ont voulu savoir ce que l'on était en train de faire à l'école des beaux-arts. Dominique nous a suggéré, à Bernard Joisten, Philippe Parreno et moi-même, de leur faire une proposition, en groupe, à plusieurs, puisque nous avions commencé à réfléchir ensemble. Tout a donc commencé par une réelle proposition d'exposition, dans un lieu assez en vue, faite à de jeunes étudiants, avec la nécessité d'essayer de faire quelque chose d'intelligent... Il est important de resituer dans son époque cette opportunité qui cristallisait notre désir : pour les étudiants d'aujourd'hui, cela ne représente plus la même chose de faire une exposition. 
Aviez-vous en tête des références d'artistes qui proposaient collectivement des expositions?

Oui, nous en avions. Avec Bernard Joisten et Philippe Parreno, nous réfléchissions à ne plus faire des œuvres comme de simples objets. Nous étions allés en 1987 à New York, où nous avions vu notamment l'exposition «The New Poverty » présentée par Collins \& Milazzo, dont la démarche, pour nous, faisait sens : des gens se mettaient à deux ou à trois pour réfléchir, ils allaient chercher des œuvres par-ci par-là, et laissaient entendre, sous un chapeau, l'identité d'une exposition, avec un discours, etc. Le résultat n'était pas forcément terrible, mais bon... Nous avons vu aussi le collectif Group Material, et plus tard General Idea à Toronto. Des collectifs mettaient en place leurs propres structures d'exposition sans se reposer sur des institutions ou des commissaires, et maîtrisaient le discours ensemble.

Ceux que vous citez pensent des formes d'exposition plutôt que des pièces autonomes. Cela engage une certaine manière de travailler ensemble.

Oui, et la commande du Magasin nous obligeait en ce sens. Et par la suite, même si parfois nous étions amenés à participer à des expositions, nous avons continué à travailler sur des propositions d'exposition dans l'exposition, ou des moments assemblés à l'intérieur d'un temps d'exposition.

Une fois la viabilité de ce type d'exposition collective avérée, y a-t-il eu des moments où vous preniez l'initiative de vous réunir pour concevoir des propositions, ou étiezvous plutôt dans la situation de rebondir sur une commande ou une invitation faite à un certain nombre d'entre vous?

Pendant deux ou trois ans, ces propositions nous étaient faites, le plus souvent, à tous. Hyper Hyper, Composit, Ozone, Siberia... c'était aux trois personnes. Au Crédac, à Ivry-sur-Seine, une proposition a été faite à Bernard Joisten, qui a demandé à faire à la fois un projet personnel et un projet à trois : Composit. Dans l'exposition se lisait un nom, celui de Bernard Joisten, puis un titre, Composit, et
« réalisé par ». Cela créait de la confusion, car on imaginait qu'il s'agissait d'un groupe qui s'appelait Ozone, Composit ou Siberia, alors que c'étaient des titres de projets.

C'est à cette époque que Philippe Thomas, après avoir été membre du collectif IFP (Information, Fiction, Publicité) que vous avez d'ailleurs côtoyé après l'école des beauxarts, a orienté son travail autour de questionnements liés à l'auteur, favorisant une mise en scène de sa disparition orchestrée dans les moindres détails.

Quant à nous, nous ne désirions pas reporter la signature sur le nom d'un groupe, mais sur le projet.

Vous travailliez collectivement, mais vous n'étiez pas un collectif...

Oui, c'était à géométrie variable. Pendant un certain temps ça a été Joisten, Parreno et moi, ensuite Joisten, Parreno et Gonzalez-Foerster, ensuite Parreno et moi, ensuite Parreno, Perrin et moi. Par la suite, Parreno a continué à collaborer avec d'autres.

Face à une prise de vue de l'exposition Composit, on a envie de savoir quel est le modus operandi : vous laissiez-vous libre, chacun, de faire ce que vous vouliez dans cette situation, tout en jetant un oil au-dessus de l'épaule de l'autre au fur et à mesure de la conception de chaque pièce? Comment discutiez-vous vos pièces? Travailliezvous au même endroit?

Pour Composit, on se considérait comme devant des écrans de Macintosh, en noir et blanc à l'époque, et on se disait qu'on travaillait avec une palette graphique, mais en les transposant à une tout autre échelle, dans l'espace d'exposition. Les briques qui composaient un fond d'écran deviennent du scotch, le fond devient de la peinture... Philippe [Parreno] avait fait de la sérigraphie en déplaçant une image comme s'il dessinait avec un pinceau, et le travail de Bernard [Joisten] devait prendre en compte le contexte, le travail de l'autre - comme une sorte de virus au milieu de l'exposition. Nous sommes d'accord sur les principes moteurs, nous nous donnons chacun une portion de l'espace. Dans ce cas précis, on ne touche pas au sol et on 
travaille sur les murs comme si l'on était en train d'utiliser une palette graphique, sauf que ce ne sont pas des électrons mais des éléments qui ont une matérialité.

Vous établissez donc un cadrage et, à partir de là, vous faites auvre commune. Ce cadrage est-il écrit?

Non, ce n'est pas écrit, c'est discuté. Par exemple, j'ai ici un collage qui a été réalisé pour Ozone (fig. 2). On pourrait dire que c'est une maquette. Je l'ai produite alors que nous ne savions pas trop encore ce que nous allions faire. Personnellement, je savais que j'allais faire des sortes de diapositives géantes et, pour les autres, j'avais imaginé tout et n'importe quoi. Par contre, ce que je savais, c'est que nous devions faire une exposition qui était comme un gaz, c'est-à-dire qui remplissait l'espace, au sol, aux murs, partout.

À l'époque, c'était vous qui utilisiez le terme de "gaz» dans ce contexte?

Oui, ensuite il est apparu ailleurs, chez Yves Michaud notamment.

Donc vous étiez tous d'accord sur ce point-là et, de votre côté, vous réalisez ce collage.

L'exposition Ozone était la troisième que nous réalisions. Ange Leccia, qui était rapporteur au FRAC Corse, nous avait fait part de l'intérêt de cette institution pour l'acquisition de l'un de nos projets. Mais ils voulaient quelque chose de nouveau et attendaient de notre part une proposition. Nous sommes partis, en réfléchissant à plusieurs, aussi avec Dominique Gonzalez-Foerster, sur des questions de nature, liées à l'écologie. Apparaissait juste à ce moment-là la question du trou dans la couche d'ozone. On avait conservé un petit article sur le sujet dans un magazine consacré à la recherche scientifique. C'est à partir de cela que l'on a gardé le nom ozone. Puis nous avons porté intérêt aux représentations que l'on pouvait relever de la nature aujourd'hui : comment nous faisait-on ressentir la nature aujourd'hui ? Avec des images liées à la science et au regard scientifique, à sa manière de capter le monde ; avec les sports fun qui commençaient à apparaître ; avec les grands cycles de disparition (on évoquait alors beaucoup les dinosaures) ; enfin, avec toutes les nouvelles technologies qui permettaient de formuler, avec des images de synthèse, des croissances de plantes, etc. Pour proposer notre projet, nous avons fait un sac, dans lequel on a mis plein d'informations, rempli de ballons, d'air, de bombes, d'une poupée de bébé, l'exemplaire du magazine Pour la science comportant l'article sur le trou dans la couche d'ozone... Et dans l'un des tubes roulés, il y avait ce collage que j'avais fait, moi, photocopié et joint à ces autres éléments. C'était un composant du projet, comme une sorte de configuration, de projection de ce que je pensais être la chose. Ce qui est intéressant, ici, c'est que le projet était choisi par Leccia à partir de ce sac qui en était l'esquisse. À aucun moment n'intervenait sa formulation. Ce n'était qu'une intention (fig. 3).

Et entre cet état et celui de l' exposition? Car il existe une photographie de cette dernière qui semble, au fond, assez proche du collage que vous nous avez montré, notamment au niveau des couleurs, de la disposition spatiale et d'une partie des objets.

Oui, même si ce ne sont pas exactement les mêmes objets. Si l'on fait une comparaison, on verra dans le collage des choses qui ne pouvaient être conservées dans l'exposition, puisque c'étaient en quelque sorte des « erreurs » par rapport à son propos. Notamment le personnage bleu : s'il y a la place pour un individu, dans Ozone, ce devrait être logiquement à travers des sièges et non à travers la représentation de la figure humaine. À l'inverse, le collage a pu nous inspirer collectivement. Ainsi, la tente qui est sur la maquette, qui devait être au départ avec d'autres objets de sport « ready-made », un habillage ou une sorte de fond décoratif de notre exposition, a été reprise dans son principe par Parreno, qui en a fait sa contribution (fig. 4).

Assez souvent, les collaborations sont aussi l'occasion de conflits et de désaccords. Ces dimensions étaient-elles présentes à cette époque?

Au début, les choses se passaient plutôt bien. 
Sans aller vers de grands conflits, arrivait-il qu' une personne signale à une autre qu'elle ne devrait pas s'y prendre comme ça? Ce qui revient à s'épauler par le jugement.

Parfois, lorsqu'une proposition était faite, tout se passait comme si les deux ou trois autres savaient ce que la troisième ou quatrième personne devait faire, qui ne correspondait pas à ce qu'elle voulait faire. Je ne dirais pas qui... mais cela arrivait. On insistait : «C'est ça qu'il faut que tu fasses, fais-le, c'est tellement évident, pourquoi tu t'emmerdes avec un tas de trucs, etc. », et finalement il le faisait. On avait très clairement en tête quelque chose à quoi nous voulions arriver. Même si c'était un projet et que l'on défendait bien l'idée que chacun fasse quelque chose de personnel, il fallait qu'à la fin cela fonctionne avec l'ensemble.

Cet « ensemble » est-il déterminé par le contexte, la réponse que l'on veut apporter à une situation, un propos général arrêté autour d'une notion? Ces éléments sont-ils ceux à partir desquels la proposition est ajustée?

Oui, par exemple pour Ozone tout le monde partageait un cadre, celui d'objets de première main, pas ou peu transformés. Quand je faisais les diapositives géantes, je suis allé demander à des producteurs d'images de synthèse, Carl Sims aux États-Unis, Untel à l'Inra... de me donner une image de ce qu'ils étaient en train de faire autour des plantes, pour Walt Disney, etc. On se reconnaissait tous dans cette logique et il ne fallait pas trop en sortir, sinon on perdait la cohérence de l'ensemble.

Votre fonctionnement collectif pourrait-il être comparé à celui d'un cabinet d'architecte, à une agence ? Vous faisait-on des contrats?

Non, le canal était très fin, cela passait par deux ou trois voies : quelqu'un nous mettait au courant d'une chose dont on lui avait parlé et pour laquelle il fallait se mettre en rapport avec Untel pour envoyer une proposition... Il n'y avait pas de marché, pas de structure, pas même d'organisation. Notre modèle collectif était beaucoup plus proche du magazine : un titre, puis des collaborateurs qui amènent des informations. Pour moi, le magazine comme source d'inspiration, c'était important. Je lisais très peu de livres à cette époque, et je pense que, tous, nous lisions très peu de livres : on regardait les magazines avant tout, les magazines anglais, français, les magazines de science... Il y avait toujours un couplage image/texte, aussi : cela nous renvoyait à une iconographie, à des images que nous n'avions pas vues. Et les expositions pouvaient se concevoir comme une mise en page. Pour Composit, par exemple, c'était vraiment une mise en page.

Votre position, qui souvent s'apparente à une réponse à une situation donnée, accorde un aspect presque stratégique à votre démarche...

Ce qui peut rester comme un point d'interrogation, c'est comment parvenir à proposer un projet alors qu'une demande a été faite ? Habituellement, on propose un projet personnel puis on essaie de le réaliser. Là, nous mettions en couple demande et projet collectif. Un jour, l'artiste Shimabuku m'a demandé si j'avais des projets personnels, c'est-à-dire hors demande, hors le fait d'être invité pour une exposition, sollicité pour telle exposition de groupe. Bien sûr, tu as un projet personnel. Mais on est parfois dans un engrenage de demandes qui fait que l'on répond aux choses, même si l'on peut intercaler l'idée d'un projet ou d'une ressaisie.

\section{De la délégation : reperformances, commandes, ateliers}

La série des "Personnages à réactiver » implique une forme de collaboration asynchrone : au-delà de la première occurrence, associant l'artiste à un performer, l'œuvre contient dans son principe même une promesse de collaboration - avec les propriétaires du personnage et ceux qui l'incarneront de nouveau. Vous avez évoqué dans un entretien la possibilité de reprendre l'un d'entre eux, "Catwoman ", avec des habits actuels, alors qu'un conservateur de musée préférera que cela se fasse avec des habits d'époque. Combien d'acteurs cela implique-t-il, et quelles décisions sont prises avec eux dans le cadre de cette collaboration? 


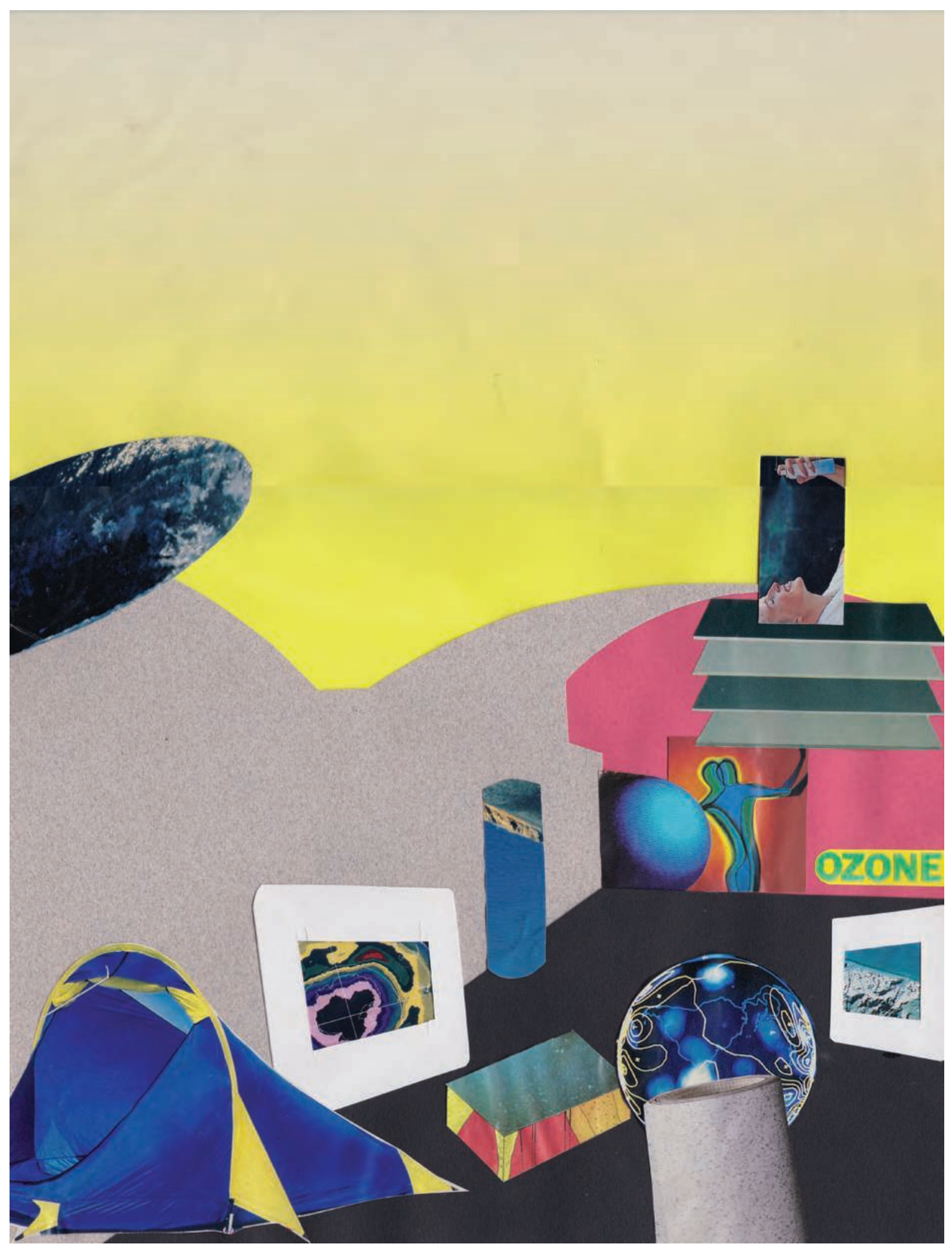

Fig. 2 : Pierre Joseph, Sans titre, 1989 ; collage original dont la photocopie est insérée dans le Sac Ozone Collection Elsa Carnielli 


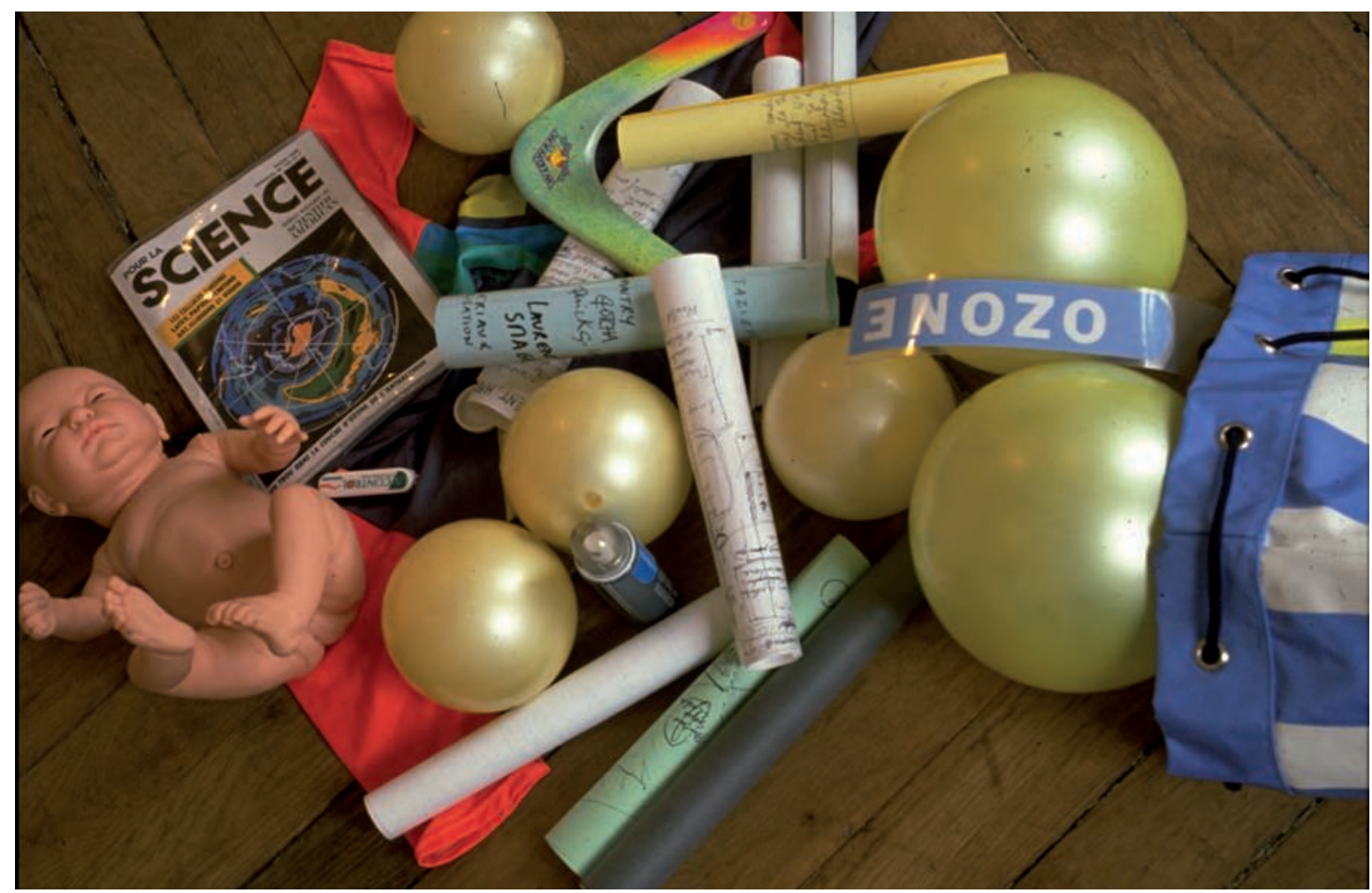

Fig. 3 : Dominique Gonzalez-Foerster, Bernard Joisten, Pierre Joseph, Philippe Parreno, Sac Ozone Version 2 , 1989 Collection Emmanuel Perrotin

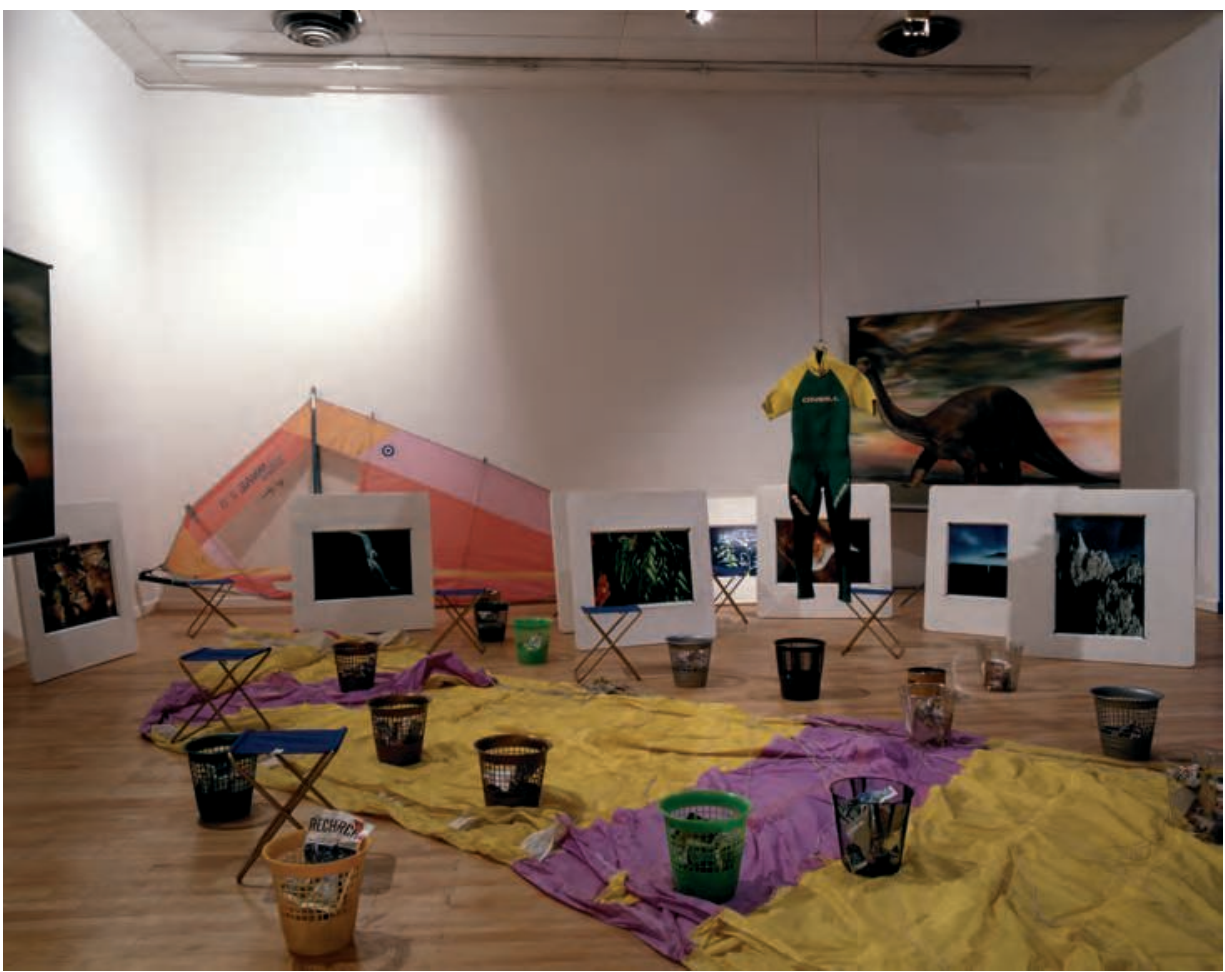

Fig. 4 : Dominique Gonzalez-Foerster, Bernard Joisten, Pierre Joseph, Philippe Parreno, Ozone, 1989; vue de l'exposition « Images, objets, scènes » Courtesy Le Magasin-Cnac (Grenoble), 1997. Crédit photographique : Le Magasin-Cnac 
Pour prendre un cas précis de personnage, celui du toréador mort, qui entretient une lointaine parenté avec celui de Manet, il a été acheté par des collectionneurs, Josée et Marc Gensollen. Ce sont eux qui ont le plus réactivé la pièce. Le collectionneur achète l'image, il achète le personnage, et il achète le droit de remontrer le personnage. Cela est noté sur un certificat, qui volontairement n'est pas trop protocolaire, pas trop directif et technique, mais souhaite laisser libre une certaine interprétation, de la même manière que j'ai, moi, été libre de l'interprétation. Les Gensollen ont donc acheté ce personnage, puis ils se sont posé la question de l'acquisition du costume. Ils avaient trouvé le costume d'un torero célèbre en vente en Espagne. Je ne disais ni oui ni non. Finalement, ils ne l'ont pas acheté : on a trouvé à chaque fois un costume en location et on restait dépendant des possibilités du moment. Je parle aux collectionneurs, je leur demande de trouver les bons objets, des choses réelles, pas des déguisements, de façon à être au plus proche. Et jusqu'à maintenant, je suis présent dans ce processus de recherche. Pour ces collectionneurs en particulier, ils ont réactivé ce personnage parfois sans moi de manière impeccable. Ensuite, j'ai reconsidéré la question du certificat, en ajoutant des précisions concernant le dernier endroit où j'ai loué le costume, en ajoutant une photographie, en précisant ce que je demandais de faire au personnage : j'ajoute une couche supplémentaire à chaque fois, un ensemble d'éléments qui ne soit pas figé, pas un certificat comme le fait Daniel Buren. Des informations, pas des contraintes, qui s'accumulent à chaque nouveau certificat. J'aime bien que cela procède par sédimentation (fig. 5).

Pouvez-vous nous parler de votre collaboration avec Mehdi Belhaj Kacem?

Je lui ai demandé de travailler avec moi dans le cadre de la proposition pour Ann Lee, le projet de Philippe Parreno et Pierre Huyghe basé sur l'exploitation par plusieurs artistes d'un personnage de manga, défini par son image et son nom, dont les droits ont été achetés à une société japonaise. Je voulais que ce personnage soit une sorte de professeur et qu'elle puisse divulguer un cours de philosophie. J'ai demandé à Mehdi Belhaj Kacem, qui venait de publier son livre Society (lui-même inspiré

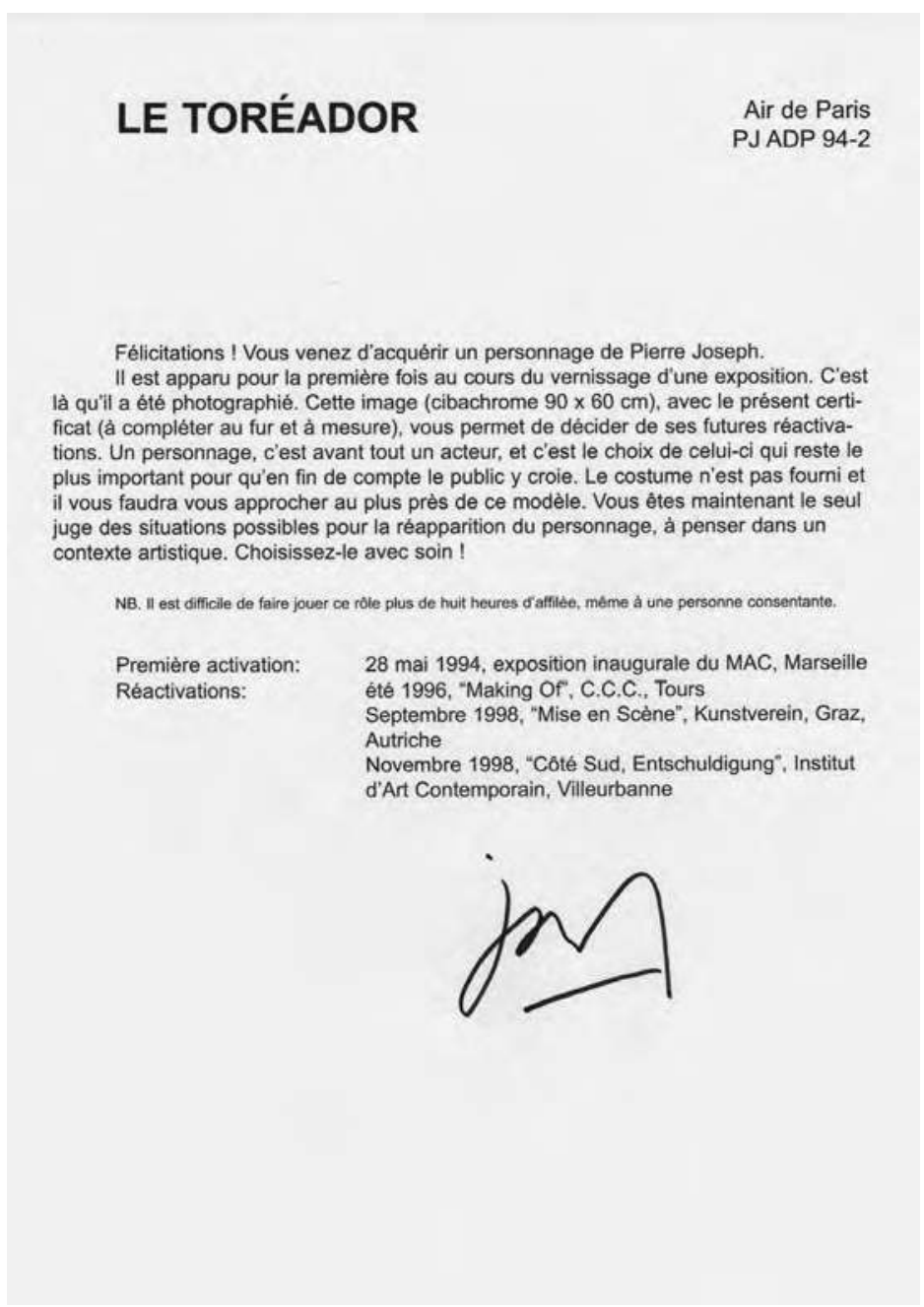

Fig. 5 : Certificat d'authenticité, Le Toréador (Personnage à réactiver), 1994 Collection Josée et Marc Gensollen

d'un jeu), s'il pouvait imaginer ce cours, comme quelque chose que l'on puisse à la fois regarder dans un centre d'art, mais aussi diffuser à l'université. Il m'a proposé un premier texte, divisé point par point : 1, 2, 3... Ça n'allait pas, je ne pouvais pas réussir à en faire quelque chose. Je lui en ai demandé un autre, et il a écrit un texte sur le jeu, la jouissance, etc., qu'il me semblait intéressant de faire jouer. On s'est mis d'accord sur une voix, sur une 
personne qui allait le dire, et sur la technologie de motion capture mise en place. Moi, c'était la forme, et lui le fond, de manière très basique. Nous avons abouti au film d'animation 3D de 34 minutes intitulé Théorie du Trickster. La première fois qu'il a été présenté, au Spot (Le Havre) en 2001, c'était dans un espace très simple, white cube. Cela marchait assez bien : on ne savait plus si on était dans un espace de prêche, de cours ou d'exposition. Cela me convenait à partir du moment où ces doutes étaient engagés : est-ce qu'on regarde une vidéo, est-ce qu'on assiste à un cours, est-ce qu'on nous prêche quelque chose ? Ensuite, au Palais de Tokyo (Action restreinte, 2003), j'ai réutilisé cette vidéo mais aussi le premier texte qu'il m'avait donné pour Ann Lee, pour une autre partie des vidéos. J'adhérais à ces textes qui venaient en décalage avec ce que je proposais, relevant d'une sorte d'indifférence aux lieux. Il y avait une sorte de netteté à faire entre un texte et ce qu'il dit et un lieu avec un fond : est-ce que ça se rejoint ou pas, est-ce que ce récit peut prendre corps dans l'espace ou non? Voilà les questions que je me posais. Assez abstraites.

Qui donnait les indications de jeu à la personne qui lisait? Qui prenait les décisions de montage?

Mehdi Belhaj Kacem était présent à l'enregistrement et c'était d'autant plus important qu'il avait fait les deux propositions de personnes pour la lecture. Il les connaissait. Nous sommes coauteurs du projet. Le texte est aussi important que l'image.

Peut-on creuser cette autre forme de collaboration consistant à travailler avec des étudiants dans le cadre d'un enseignement, particulièrement lorsqu' elle aboutit à une ouvre, à l' exemple de ces dessins réunis dans un livre, Atlas? C'est un type de collaboration particulier, d'autant plus qu'il est pris dans un cadre pédagogique (fig. 6).

Cela fait partie d'un exercice, d'un protocole. Ce qui est important, c'est la commande, la décision. Si je fais un raccourci, c'est un peu comme en sociologie, où les professeurs demandent à leurs étudiants d'aller poser des questions, les récupèrent et font la synthèse. À la différence que j'avais énoncé clairement ce que j'allais faire avec leurs tra- çages. Cela réunissait des étudiants de première année des écoles des beaux-arts de Nice, Montpellier, Avignon, Sète. Le protocole était celui d'un workshop. Qui dit workshop dit sous la coupe d'un artiste qui dirige et qui va demander quelque chose aux étudiants : l'accrochage d'une exposition, une réalisation... Pour ce workshop, leur force de travail, c'était le traçage, leur mémoire et leurs dessins. L'idée du workshop, c'était de dessiner de mémoire le cosmos, le planisphère, la carte de son pays d'origine, la carte de sa ville, le système digestif, enfin l'appareil sexuel féminin/ masculin en coupe et en vue anatomique. Donc, on passait du plus global à l'intime. On demandait cinq dessins, cinq versions successives, afin de s'approcher le plus possible du modèle que l'on avait en tête. Puis on demandait de nous redonner les cinq approches.

Les étudiants se sont déplacés à Sète, à côté de l'école, pour participer. Ils découvraient le protocole à leur arrivée. Ils ont dessiné, chacun, puis on a regardé les dessins, ce qui permettait de voir où l'on en était de ces représentations, de ce que l'on est censé savoir, de ce que l'on a appris. J'ai fait signer un papier aux étudiants - qui à mon avis ne doit avoir aucune valeur juridique - autorisant toute utilisation de leurs dessins avec leur nom et leur signature. Mais au fond, ils s'en défaisaient facilement, comme cela était un exercice et qu'ils n'y inscrivaient pas leur autorité. Ensuite, j'ai fait des agrandissements de ces dessins pour qu'ils fonctionnent tous ensemble. À la fin de l'ouvrage, on peut trouver une liste de noms qui renvoie aux différents dessins, numérotés.

Cela évoque une forme traditionnelle d'atelier, avec une tâche à accomplir collectivement, cadrée du début à la fin par un maître qui délègue sa main.

Oui, il y a atelier dans ce sens-là.

Il est un aspect de cette collaboration que vous n'avez pas restitué dans le livre : la discussion/confrontation finale, lorsque vous compariez les dessins.

Cette conclusion, je ne la voulais pas dans l'œuvre. Celui qui regarde le livre peut la faire de lui-même. Mais il était intéressant de la faire avec les étudiants, dans un second temps du travail. 

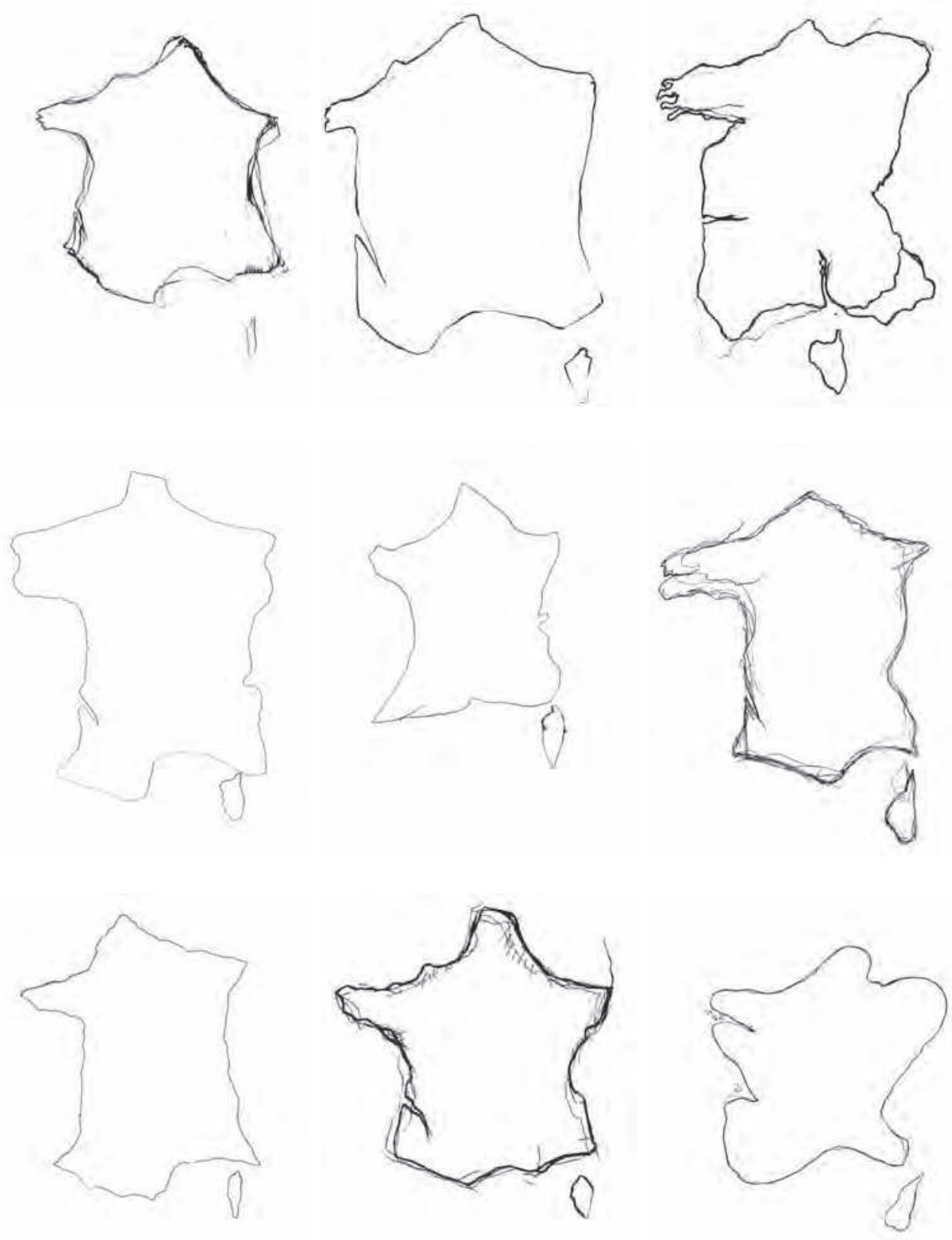

Fig. 6 : Neuf dessins extraits de Pierre Joseph, Atlas (La carte de la France), publié dans Atlas, 2004, Montpellier, galerie chantierBoîteNoire. Recueil des 480 dessins du projet Atlas, texte de Christian Besson ; édition 20 ex. 
L'objectif aurait pu être de considérer ce que fait cette expérience chez les gens qui la font. Ce que vous avez rete$n u, i c i$, c'est le dessin.

Ce qui m'intéresse, c'est l'ouverture des lacunes : passer d'un imaginaire ou d'un irrationnel à du savoir. Heureusement que l'on transforme tout, que l'on revoit tout, que l'on digère toutes ces représentations. C'est cet écart qui m'intéresse.

Quels autres types de collaboration abordez-vous aujourd' hui ?

Je fais des projets avec ma compagne, Elsa Carnielli. Elle m'a aidé sur des commandes publiques ou des $1 \%$. Et je me dis que le prochain projet, on le signe à deux, en raison du travail et de la façon dont se mettent en place les choses. C'est une manière de clarifier les choses. On ne se présente pas de la même façon, on ne revendique pas les choses de la même façon. Il y a tellement d'acteurs et de compétences différentes autour de ces réalisations publiques que je préfère de loin une autorité partagée.

Nicolas Donin est musicologue à l'Institut de recherche et de coordination acoustique/musique, où il dirige l'équipe Analyse des pratiques musicales (Ircam-CNRS-UPMC). Il est l'auteur d'études sur les processus de création musicale, l'histoire des pratiques d'écoute et d'analyse musicale depuis la fin du XIXe siècle, et l'analyse musicale d'interprétation, publiées à la fois dans le champ musicologique et dans celui des sciences humaines. Il est coauteur de courts-métrages, de DVD-Roms et de publications en ligne issus de ces recherches.

Nicolas.Donin@ircam.fr

Christophe Kinm est critique, commissaire d'exposition indépendant et professeur à la Haute école d'art et de design de Genève. Ses écrits critiques et théoriques portent en majorité sur les arts visuels, la musique et l'esthétique, et furent publiés dans des ouvrages collectifs, catalogues d'exposition et revues. Ses recherches récentes ont été consacrées aux pratiques artistiques de l'archive, aux figures et méthodes de la transmission en art et aux processus d'improvisation dans les sciences sociales et les pratiques artistiques. Il a publié, en 2013, L'Épreuve de l'image, techniques et compétences des corps, aux éditions Bayard (coll. « Logique des images »). 